\title{
Surface Mapping of Motor Points in Biceps Brachii Muscle
}

\author{
Ja-Young Moon, M.D., Tae-Sun Hwang, M.D., Ph.D. ', Seon-Ju Sim, DDS., Ph.D. ${ }^{2}$, \\ Sae-il Chun, M.D. ${ }^{3}$, Minyoung Kim, M.D., Ph.D. ${ }^{3}$ \\ Department of Physical Medicine and Rehabilitation, Eunpyeong Hospital, Seoul 122-913, \\ ${ }^{1}$ Department of Anatomy, School of Medicine, CHA University, Departments of ${ }^{2}$ Dentistry, \\ ${ }^{3}$ Rehabilitation Medicine, CHA Bundang Medical Center, CHA University, Seongnam 463-070, Korea
}

Objective To localize the site of motor points within human biceps brachii muscles through surface mapping using electrophysiological method.

Method We recorded the compound muscle action potentials of each lattice of the biceps brachii in 40 healthy subjects. Standardized reference lines were made as the following: 1) a horizontal reference line (elbow crease) and 2) a vertical reference line connecting coracoid process and mid-point of the horizontal reference line. The Compound muscle action potentials were mapped in reference to the standardized reference lines. The locations of motor points were mapped to the skin surface, in the ratio to the length of the vertical and the half of the horizontal reference lines.

Results The motor point of the short head of biceps was located at $69.0 \pm 4.9 \%$ distal and $19.1 \pm 9.5 \%$ medial to the mid-point of horizontal reference line. The location of the motor point of the long head of the biceps was $67.3 \pm 4.3 \%$ distal and $21.4 \pm 8.7 \%$ lateral. The motor point of the short head of the biceps was located more medially and distally in the male subjects compared to that in the female $(\mathrm{p}<0.05)$.

Conclusion This study showed electrophysiological motor points of the biceps brachii muscles through surface mapping. This data might improve the clinical efficacy and the feasibility of motor point targeting, when injecting botulinum neurotoxin in biceps brachii.

Key Words Motor point, Surface mapping, Biceps brachii, Botulinum toxin, Motor point block

Received January 5, 2011; Accepted May 9, 2011

Corresponding author: Tae-Sun Hwang

Department of Anatomy, School of Medicine, CHA University, 222, Yatap-dong, Bundang-gu, Seongnam 463-070, Korea

Tel: +82-31-725-8303, Fax: +82-31-725-8244, E-mail: hwangtaesun@cha. ac.kr

(a) This is an open-access article distributed under the terms of the Creative Commons Attribution Non-Commercial License (http:// creativecommons.org/licenses/by-nc/3.0) which permits unrestricted noncommercial use, distribution, and reproduction in any medium, provided the original work is properly cited.

Copyright $\odot 2012$ by Korean Academy of Rehabilitation Medicine

\section{INTRODUCTION}

Motor point blocks or nerve blocks using phenol or botulinum toxin-A are widely used to improve abnormality of gait, posture disorder, loss of ability to perform activity of daily living and voiding dysfunction induced by spasticity in stroke, cerebral palsy, and spinal cord injury. ${ }^{1,2}$ Phenol can relieve the characteristic symptom of spasticity, such as exacerbated stretch reflex or hypertonictiy of 
muscle by inhibiting stretch reflex arc through the nerve degeneration and is expected to produce remarkable effect in spite of the cheap price in comparison with the other methods of relieving spasticity. Further, it has the advantage of a relatively long lasting and selective effect. However, if it was not injected on the motor point exactly, the therapeutic effects for the spastic disorder could be reduced and cause side effects, such as unwanted muscle weakness, abnormal sensations and pain. Meanwhile, the botulinum toxin used popularly in recent practices, which is the type-A neurotoxin, that is generated in the clostridium botulinum and can cause muscle paralysis by blocking neurotransmission with the irreversible inhibition of the release of acetylcholine in presynaptic membrane of myoneural junction. Its effect is known to last 4 to 12 weeks. Shaari and Sanders ${ }^{3}$ reported that the injection of toxin in the site of $5 \mathrm{~mm}$ far away from the motor endplate showed a $50 \%$ reduction of denervation and paralysis compared with the injection of botulinum toxin into neuromuscular junction in a rabbit model. Childers et al. ${ }^{4}$ reported that the average muscle strength, at two and five weeks, started to drop more in the injection of botulinum toxin in the motor endplate using electromyography (EMG) than compared to the injection without EMG in canine models. So now we know that even in case of the botulinum toxin, it is necessary to make the injection on the accurate motor point.

Motor points are elecrophysilogically defined, as the point with the highest excitability of the muscle or the point on the skin where muscle contraction can be observed by the least electrical stimulation or the point on the muscle where muscle contractions can be caused by the minimum intensity and short duration electrical stimulation. Which, anatomically, is defined as the area where motor endplates (terminal area of the motor nerve fiber) are dense. ${ }^{1,5,6}$ Until recently, the studies to find the location of the motor points were mainly cadaver dissection. In these cases, the location of the motor points were defined as the area where motor nerve enter into the muscle and can be verified with the naked eye. ${ }^{7-12}$ However, since anatomically motor endplates are formed after multiple small motor nerve branches, which originate from the large myelinated motor nerve axon accessing to the muscle, travel along the surface of myofiber in regular short distances, ${ }^{8}$ the determination of the position through the cadaver dissection study could have limitations in locating the motor points for botulinum toxin injection. In this study, we thought that for an easy access of the motor points, objective and standardized surface mapping on the skin was necessary. So we defined motor points of the biceps brachii muscles, as the area where the maximum amplitude appeared, when we recorded the evoked compound muscle action potentials from the biceps brachii, with anteromedially stimulating the musculocutaneous nerve at the proximal $1 / 3$ position of the humerus using electromyography. In addition, through the surface mapping of the biceps brachii muscles that could have an effect on the activity of daily living, we would like to help in setting-up the proper location of injection, especially when botulinum toxin is injected.

\section{MATERIALS AND METHODS}

\section{Materials}

There were 80 upper limbs (40 right, 40 left) from 40 healthy adults (male 20, female 20) who have not had an injury or surgery which could affect the nerve distribution of the biceps. The average age was 43.4 years and the average height was $165.3 \mathrm{~cm}$. This study has passed the review of Institutional Review Board (IRB) of Eunpyeong hospital. The purpose, research method and significance of this study were explained to all the enrolled subjects and thereby, obtaining their consents, after they have been described to them, in detail.

\section{Methods}

The subjects were placed at shoulder $45^{\circ}$ abduction, elbow extension and forearm external rotation in supine position. For mapping of the motor points on the skin surface, standardized reference lines were made. The horizontal reference line was defined as the line connecting both end points of the elbow crease in $90^{\circ}$ flexion of the elbow. The vertical reference line was defined as the line connecting the tip of coracoid process and the midpoint of the horizontal reference line.

Using Medelec Synergy EMG machine (Oxford instrument Co., Surrey, UK), we recorded the evoked compound muscle action potentials from the biceps brachii, after anteromedially stimulating the musculocutaneous nerve at the proximal $1 / 3$ location of the humerus (the length of humerus was defined as the length between acromion and lateral epicondyle). The electrical stimulation and output of the potentials were done by a physiatrist and the recording was done by a technician. We recorded 


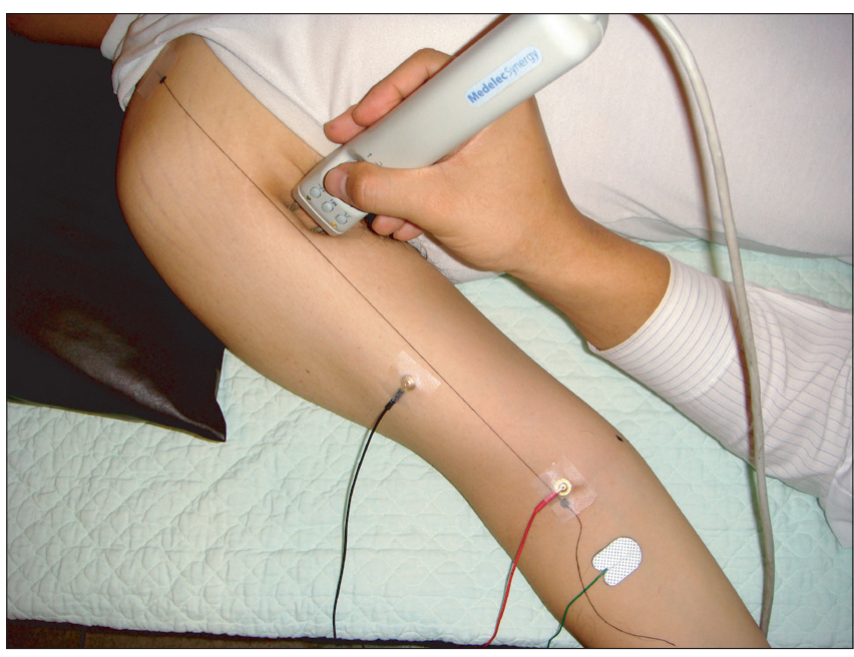

Fig. 1. Motor point mapping method. The coracoid process and end points of the elbow crease in $90^{\circ}$ flexion of the elbow were identified as reference points. Standardized reference lines were made as a horizontal reference line drawn between both end points of the elbow and a vertical reference line drawn between mid-point of the horizontal reference line and coracoid process. Stimulating electrode was located on musculocutaneous nerve at the proximal $1 / 3$ area on anteromedial surface of the arm. Recording surface electrode was located on the long head of biceps brachii muscle area.

the compound muscle action potentials (CMAPs) using discoid recording electrodes that were $1 \mathrm{~cm}$ in diameter. This recording was done after a stimulation with constant supramaxiamal strength (50-70 $\mathrm{mA})$ once per second, while stimulating the surface electrodes that are placed in deeply and perpendicular to the skin, as near as possible to the musculocutaneous nerve.

We defined the peak of coracoid process as an original point and we set the vertical and horizontal coordinates of $1 \mathrm{~cm}$ intervals in the right and left side from the vertical reference line, from which, the CMAPs of the biceps brachii were recorded (Fig. 1). Comparing the recorded CMAPs, we moved the recording electrodes left and right, up and down at intervals of $0.5 \mathrm{~cm}$ from the coordinate where the largest CMAPs were recorded. We then defined the area where maximal amplitude was recorded as the motor point. The distance from the vertical reference line to the motor point was recorded as an $\mathrm{x}$ value and the distance from the coracoid process to the point where the perpendicular line crossed the vertical reference line was defined as a y value. $x$ and $\mathrm{y}$ values were relative values, which were calculated as percentages ( $x$ value; to the half of the length of the hori-

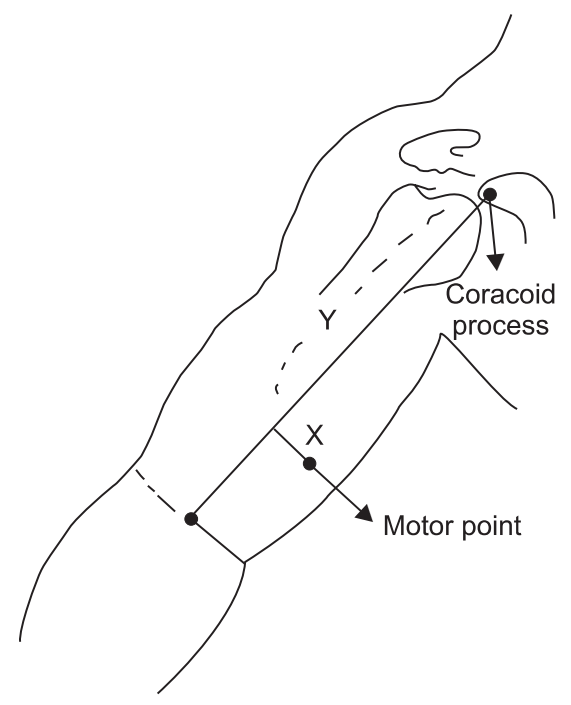

Fig. 2. Diagrammatic drawing of measurement of the location of the motor point of the biceps brachii muscle. The distance from the vertical reference line to the motor point was recorded as an $\mathrm{x}$ value and the distance from the coracoid process to the point where the perpendicular line crossed the vertical reference line was defined as a y value.

zontal reference line, y value; to the length of the vertical reference line) (Fig. 2).

\section{Experimental analysis of the data}

The mean and standard deviation of all the indicators were calculated. Further, using an independent t-test, the position of the motor point in male and female cases was compared. Significance level was $\mathrm{p}<0.05$.

\section{RESULTS}

1. The motor point of the short head of biceps brachii was confirmed in the medial side from the vertical reference line. It was located at the intersection of $69.0 \%$ distal from coracoid process and $19.1 \%$ medial to the mid-point of the horizontal reference line. The motor point of the long head of the biceps brachii was confirmed in the lateral side from the vertical reference line. It was located at the intersection of $67.3 \%$ distal and $21.4 \%$ lateral (Table 1) (Fig. 3).

2. In comparison, between males and females of the motor point of biceps brachii, the motor point of the short head of the biceps was located more medially and distally in males compared with that in females. However, there was no statistically significant difference in the motor point of the long head (Table 2). 
3. There was no difference with statistical significance between the left and the right side of the motor point of biceps brachii (Table 3).

\section{DISCUSSION}

In patients with stroke, cerebral palsy, cervical spinal cord injury, increased spasticity of elbow flexor may cause adverse effect on daily performance and walking balance. To solve this spasticity of the elbow flexor, several treatment methods have been developed, for example, physical therapy, orthotics, medication, and injection therapy and so on. These injection methods, used commonly, are nerve branch or motor point block using phenol and motor point (neuromuscular junction) block using botulinum toxin, which is used popularly in recent practices. ${ }^{9,13-19}$

Table 1. Mean Distance of Motor Points of the Biceps Brachii Muscles from Reference Lines

\begin{tabular}{lcc}
\hline & Short head & Long head \\
\hline Vertical distance* $(\%)^{\dagger}$ & $69.0 \pm 4.9$ & $67.3 \pm 4.3$ \\
Horizontal distance $^{\dagger}(\%)$ & $19.1 \pm 9.5^{\ddagger}$ & $21.4 \pm 8.7^{\S}$ \\
\hline
\end{tabular}

Values are mean \pm standard deviation

*From the coracoids process to the point where the perpendicular line, crossed the vertical line. \%; Percent of the arm length. ${ }^{\dagger}$ From the vertical reference line to the motor point. \%; Percent of the half of the arm width. ${ }^{\ddagger}$ Medial side to vertical reference line. ${ }^{\S}$ Lateral side to vertical reference line
In Korea, prior to the studies of the motor points of the upper arm, the research for describing the motor points of gastrocnemius and soleus muscles have been reported. Kim et al. ${ }^{7}$ reported that the first motor point of the medial gastrocnemius muscle was located at $5.0 \%$, the second was at $16.0 \%$, the first motor point of lateral gastrocnemius was located at $10.0 \%$, the second was at $16.0 \%$, the first motor point of soleus muscle was located at $18.0 \%$ and the last motor point at $19.0 \%$ of the lower leg length. Further, Kim et al. ${ }^{8}$ reported that the first motor point of the medial gastrocnemius muscle was located at

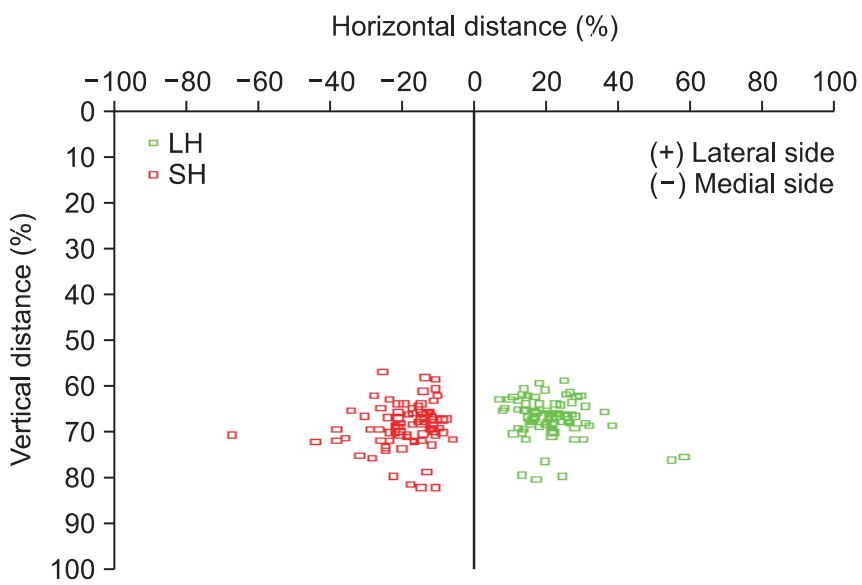

Fig. 3. A plot of the location of the motor points in the biceps brachii muscles. $x$ value is the distance from the vertical reference line to the motor point, $y$ value is the distance from the coracoid process to the point where the perpendicular line crossed the vertical reference line. SH: Short head, LH: Long head.

Table 2. Mean Distance of Motor Points of the Biceps Brachii Muscles from Reference Lines in Male and Female Cases

\begin{tabular}{llclcc}
\hline \multirow{2}{*}{ Sex (cases) } & \multicolumn{2}{c}{ Short head (\%) } & & \multicolumn{2}{c}{ Long head (\%) } \\
\cline { 2 - 3 } \cline { 5 - 6 } & \multicolumn{1}{c}{$\mathbf{X}^{*}$} & $\mathbf{Y}$ & & $\mathbf{X}^{\dagger}$ & $\mathbf{Y}$ \\
\hline Male (40) & $22.9 \pm 10.9^{\ddagger}$ & $70.5 \pm 4.8^{\ddagger}$ & & $21.9 \pm 10.4$ & $68.2 \pm 4.3$ \\
Female (40) & $15.3 \pm 5.9$ & $67.5 \pm 4.6$ & & $20.9 \pm 6.7$ & $66.4 \pm 4.0$ \\
\hline
\end{tabular}

Values are mean \pm standard deviation

${ }^{*}$ Medial side to vertical line, ${ }^{\dagger}$ Lateral side to vertical line, ${ }^{\ddagger} \mathrm{p}<0.05$ : Comparison between male and female

Table 3. Mean Distance of Motor Points of the Biceps Brachii Muscles from Reference Lines in Right and Left Sides

\begin{tabular}{cccccc}
\hline \multirow{2}{*}{$\mathbf{0}$} & \multicolumn{2}{c}{ Short head (\%) } & & \multicolumn{2}{c}{ Long head (\%) } \\
\cline { 2 - 3 } \cline { 5 - 6 } & $\mathbf{X}^{*}$ & $\mathbf{Y}$ & & $\mathbf{X}^{\dagger}$ & $\mathbf{Y}$ \\
\hline Rigt (40) & $19.9 \pm 10.5$ & $68.7 \pm 4.5$ & & $20.7 \pm 9.0$ & $66.7 \pm 4.2$ \\
Left (40) & $18.3 \pm 8.4$ & $69.3 \pm 5.3$ & & $22.0 \pm 8.5$ & $67.9 \pm 4.3$ \\
\hline
\end{tabular}

Values are mean \pm standard deviation

*Medial side to vertical line, ${ }^{\dagger}$ Lateral side to vertical line 
$9.6 \%$, the last at $37.5 \%$ and the first motor point of lateral gastrocnemius was located at $12.0 \%$, the last was at $37.9 \%$ and the first motor point of soleus muscle was located at $20.5 \%$, as well as the last motor point at $46.7 \%$ of the lower leg length. The reason why there are differences in the results of both studies may be associated with the different definition of the motor point in the two researchers. Kim et al. ${ }^{7}$ defined the motor point as the area where the motor nerve branch enters into the muscle (macroscopic entry into the muscle), however Kim et al ${ }^{8}$ defined the motor point as the point where the intramuscular motor nerve branch was tracked so deep that the branch could not be separated from the muscles (microdissection). Kim et al. ${ }^{8}$ differentiated their definition because of the fact that anatomically, the motor point were dense regions of the motor endplates, which were the end regions of the motor nerve fiber and the motor endplates were formatted after a big myelinated motor nerve axon that approached the muscle was divided into multiple smaller branches, and which traveled along the surface of the muscle for a certain distance. They also considered that the location of the motor point in observing anatomically, where the motor nerve branch enters into the muscle with the naked eyes, was different from that of the motor point in the electrical diagnostics. In clinical practice, the motor point could be found by inserting the needle into the muscle, where maximum muscle contraction can be caused by the minimum stimulus after searching the region of the response that was the most remarkable in electrical stimulation on the skin surface around the area, which has the most tension in palpation or the area where the muscle seemed swollen visually or around the standard insertion area of the needle EMG. But so far, there has not been any specific method for practicing or any quantitative standard for positioning of the motor point. ${ }^{20}$ So Ko et al. ${ }^{20}$ attempted the objective and standardized mapping on the surface of the skin via electrical stimulation for easy access of the motor point. Ko et al., ${ }^{20}$ using EMG devices, stimulated the posterior tibial nerve electrically at the popliteal area and recorded the evoked compound muscle action potentials of triceps of the lower leg and defined the motor point as the area where the maximum amplitude appeared, and then they reported that the motor point of the medial gastrocnemius muscle was located at $41.0 \%$, the lateral gastrocnemius at $35.7 \%$, the soleus at $68.6 \%$ of the lower leg length from proximal to distal. This result showed that the discrepancies of the location of the motor point exist between in the cadaver dissection study and in the surface mapping study. We think that this result show that the motor point of microdissection study, in which the motor point was described after tracking the motor nerve branch as much as possible, could be closer to the location of the motor point based on the anatomic definition - the crowded area of the motor endplates, which are the end of motor nerve fiber - rather than the motor point in the study in which motor point was defined as the first area for the motor nerve branch to enter into the muscle. Furthermore, we think that the result suggests that the study method of Ko et al. ${ }^{20}$ may be a more reasonable way to find the dense area of the motor endplates, rather than the cadaver dissection studies. In addition, we think it is necessary to vary the access to the motor point, according to which block is performed in the phenol or botulinum toxin. As such, we think that in the case of phenol block, it is reasonable to refer to the location of the motor point in the earlier cadaver studies, since we believe that when practicing the whole nerve block, the motor branch block, the motor point block using phenol, ${ }^{16}$ according to the patient's conditions, both the first point for the motor nerve to enter into the muscle (macroscopic entry into the muscle) and the point for intramuscular branch to enter into the muscle after being tracked maximally (microscopic entry into the muscle), are expected to produce some sufficient clinical effects. In other words, the motor point, in the cadaver dissection study (the first area for the motor nerve or motor nerve branches to enter into the muscle), which is thought to be located proximally to the motor endplates, is a valid position for phenol block. Clinically, we believe that in phenol block, the nerve conduction devices using EMG needle is necessary. However, this information in the cadaver dissection study can provide the approximate information about the place in which to locate the needle first and this can help to reduce the pain of the patients in addition to giving a time advantage to the practitioners through avoidance of the repeated sticking and make better therapeutic effects. However, we thought that in contrast with motor point block using phenol, crowded areas of the motor endplates that is the target in motor point block using botulilum toxin, could be different from the location of the motor point in the cadaver dissection study. Further, the method by Ko et al., ${ }^{20}$ in which the surface mapping of the motor points were performed using the surface nerve 
stimulation, could be more of a reasonable method for reflecting the ultimate conditions of the motor endplates. Therefore, we performed our study.

In this experiment, when the vertical length of the upper arm was defined as the length of the line connecting coracoid process and mid-point of the elbow crease along with the horizontal length of the upper arm (upper arm width) as the length of the elbow crease, the motor point of short head and long head of biceps brachii was located at about $2 / 3$ of the vertical length from coracoid process, respectively, and about $1 / 5$ of the half of the horizontal length from the vertical reference line on both sides. When the results of our study compared with the results of other studies, consideration of the disagreement of the motor point might be needed, as in the study of the motor point of the lower leg. In the cadaver dissection studies, when the motor point was defined as the area for the motor nerve or motor nerve branch to enter into the muscle (macroscopic entry into the muscle), Buchanan and Erickson ${ }^{9}$ described the motor point of the biceps brachii near $1 / 2$ of the upper arm (53\%) in 13 cadaver, 26 arms. Further, Kim et al. ${ }^{10}$ reported in the study of 5 cadaver, 9 arms, that the motor points of the biceps brachii were distributed at about $1 / 2$ (first motor point of the short head was located at $47.5 \%$, the second $51.8 \%$, the first motor point of long head was located at $53.0 \%$, the second $57.7 \%$ ) of the upper arm. In addition, Park et al. ${ }^{11}$ also reported that the motor point of biceps brachii was observed at about half of the upper extremity (short head $48.24 \%$, long head $53.19 \%$ ). In our opinion, as in the studies of the motor point of the lower leg, the location of the motor points described in these three studies could be appropriate in the motor point block using phenol. Unlike earlier studies, Lee et al. ${ }^{12}$ investigated the area where the points for the motor nerve branches to enter into the muscle (intramuscular motor point) using micro-dissection, were most densely distributed, as well as the area for the motor nerve entering into the muscle (motor entry point) in 30 cadavers, 56 upper limbs. In biceps brachii, they reported that in relation to a reference line, connecting the coracoid process and the medial epicondyle of humerus, the motor entry point was located at $46.1 \%$ (short head $44.3 \%$, long head $47.9 \%$ ) and intramuscular motor points were most densely distributed at a length from $64.6-70.3 \%$ from the coracoid process, these results closely match our results. Furthermore, Lee et al. ${ }^{12}$ determines the location of the motor endplates through the micro-dissection, but did not confirm by nerve staining.

In addition to the cadaver dissection, there have been attempts to locate the motor endplates of biceps brachii through histological methods. Aquilonius et al. ${ }^{21}$ studied the location of the motor endplates of the biceps brachii, through the histological method (whole mount cholinesterase staining), and have reported that the endplates were primarily distributed as a $5-10 \mathrm{~mm}$ wide band in a length of 4-6 cm long region that was halfway between the tendons of the muscle. Amirali et al. ${ }^{22}$ localized the motor endplate of biceps brachii through the histological method (3 muscles processed by sihler's stain, 2 muscles by acetylcholinesterase stain) and reported that the major motor endplate region was distributed as an inverted Vshaped band, $1 \mathrm{~cm}$ in width at about $2 / 3$ of the length of a reference line connecting the acromion and olecranon, the results are similar to those of our study. Studies of the muscle fiber and the location of the motor endplates have been performed mainly through histological methods, Coërs $^{23}$ reported that in young children's muscle, motor endplates gathered in the narrow band shape and this motor endplate was always located in the middle of the origin and the insertion point of the muscle fiber, in addition, the shape of this band depends on the texture of the muscle and how the muscle fibers insert, so when the muscle fibers were inserted into the narrow tendon, such as biceps brachii, the band formed more or less a shape of a parabola. In addition, Using histological methods used in the study of Coërs, Christensen ${ }^{24}$ conducted the study in stillborns and reported that the direction of the muscle fiber was up to the muscle fiber length and pennation, therefore, in the unipennate muscle, motor endplates formed striped shape in the middle of the muscle, in the bipennate muscle, formed the concave endplates belt shape and the multipennate muscle such as sartorius or gracilis muscle, formed scattered pattern. Biceps brachii muscle was actually two separate unipennate muscles, with fibers running through the whole length of the muscle, except in the medial distal part of the short head some shorter fibers exist, and the endplates form a distinct band through the middle of the two heads, in the short head a smaller endplate band was seen in the distal part of the muscle. Further, Deshpande et al. ${ }^{25}$ predicted theoretically the position of the motor endplate by considering pennation, location and orientation, and that in biceps brachii muscle, which was a unipennate 
fusiform muscle, the motor endplate should form an inverted V-shaped band, slightly below the middle of the humerus. In addition, with respect to the motor endplate of the short and long head, the endplate band of the long head would be a little higher than that of the short head because the fibers of the short head begin a little below and end slightly above that of the long head. This result is consistent with that of our study.

In addition, there have been studies that analyzed the myoelectric signal, which was detected via surface electrodes, when muscle was contracted. Masuda et al. ${ }^{26}$ conducted analysis of myoelectric signal from the biceps brachii of 3 subjects, using surface electrode array. They arranged stainless steel, strip electrodes in a $25 \times 4$ configuration on an acryl plate, which was molded to fit the shape of the right biceps brachii. Each electrode was $1 \mathrm{~mm}$ in diameter and $10 \mathrm{~mm}$ in length, and they were separated by $5.0 \mathrm{~mm}$ in the longitudinal direction and 2.5 $\mathrm{mm}$ in the lateral direction. They analyzed myoelectric signal of the biceps brachii that occurred when the force of $40 \%$ of the maximum voluntary contraction was given. The neuromuscular junctions of the biceps brachii were located nearly at the middle length of the muscle and distributed in the zone. In 1 subject, neuromuscular junctions occupied a single zone, across the muscle, while the other 2 subjects had 2 parallel zones separated by 10-20 $\mathrm{mm}$. By a similar method, Saitou et al. ${ }^{27}$ investigated the distribution of innervation zones of the biceps brachii in 3 subjects by detecting bi-directional propagation of the motor unit action potentials (MUAPs) with the multichannel surface electrode array and they reported that the innervation zones were distributed in a narrow band around the muscle belly. In one subject, the innervation zones in the short head were scattered in a wider band.

In our study, motor point of the short head had slightly more distal position than that of the long head. This matches the prediction of Deshpande et al. ${ }^{25}$ However, this is slightly different from the results of Amirali et al., ${ }^{22}$ who showed that the motor endplate band was located at $75 \%$ at the lateral edge, $61 \%$ at the midline, and $72 \%$ at the medial edge when the length of the upper arm was defined as the distance between acromion and olecranon. In regards to the cause of this discrepancy, future research needs to be conducted. In comparison between male and female, the motor point of the short head of the biceps was located more medially and distally, in the male when compared to that in the female. This result might be related to the fact that women have more fat tissue and less muscle tissue than men. However, further study is thought to be needed to confirm this. In our study, when we made a horizontal reference line and a vertical reference line for the surface mapping of motor points in the biceps brachii muscle, we defined the length of the upper arm as the distance between coracoid process and mid-point of the elbow crease. The reason why we set the elbow crease in $90^{\circ}$ flexion of the elbow, as the horizontal reference line, was that this could be detected easily in the supine position, and we thought that this could be useful, especially in patients whose arm had flexor synergy, which could make accurate identification of anatomic landmarks technically difficult. The vertical reference line was defined as the line connecting the mid-point of the horizontal reference line and coracoid process since the tip of coracoid process was easier to palpate than the acromion, and a setting like this could help more easily to display two-dimensional location of the motor points, with respect to the injection point of botulinum toxin through the easier distinguishing of the short head and the long head of the biceps brachii. Further, the reason why we used the proportional distance, when setting the coordinates of the motor point, was that we wanted for the results of this study to be available in both groups of adults and children, as it was known that motor innervations was completed in the early childhood days and the location of motor endplates in the adult muscle was correlated with that in the pediatric muscle. ${ }^{21,23,24}$ In addition, with regards to the stimulating area, the reason why we stimulated the musculocutaneous nerve at above $1 / 3$ of the arm length was that this area would be the most appropriate area to stimulate the nerve, when considering the mileage of musculocutaneous nerve, which exited the brachial plexus then pierced the coracobrachialis muscle then passed between biceps brachii muscle and brachialis muscle then at $1 / 3$ of the arm length, which then produced the first branch to the biceps brachii at $45 \%$ of the arm length. ${ }^{28}$ In addition, this area was known to be the main route for the block of musculocutaneous nerve. ${ }^{29}$ Meanwhile, with regards to erb's point, we thought that it was inappropriate in our study, since the subjects could not bear such repetitive and strong stimulations in that area.

Thus, with regards to the total mileage of musculocutaneous nerve, when putting together the findings of the cadaver dissection studies, the histologic studies, and our 
study, we could summarize this as the following; after the musculocutaneous nerve came out of coracobrachialis muscle, the nerve branch to the biceps brachii would exit from nerve trunk at about $45 \%$ of the arm length ${ }^{28}$ then, at about $50 \%$ of arm length, would reach the motor point of the biceps brachii, which could be described as a macroscopic entry into the muscle in the cadaver dissection studies. ${ }^{9-12}$ Then the neuromuscular junctions would be formed at near $2 / 3$ of the arm length, which results were showed in our study and also could be compatible with that of the histologic studies. ${ }^{21-24}$ Furthermore, this position could be regarded as the target area for motor point block using botulinum toxin. When practicing the method of motor point block ${ }^{30}$ with conventional electromyography equipment, we stimulate target muscle directly, using only cathode with anode fixed to tendon and found a place to produce maximum twitch with the minimum stimulus, then block such points. However, although the actual neuromuscular junctions may be located near the motor point, it will be difficult to say that they are exactly located under the motor point, for actually they are present on each muscle fibers and are spread throughout the muscle. ${ }^{26}$ Although finding the location of the motor endplate using electromyography might be possible through observing the spontaneous activity with the characteristic shapes (end-plate activity), this method could be time consuming and ensure great pain to patients. In clinical practice, although the results of our study could not substitute for the motor point block with electromyography, it could be contributed to increase the efficiency and the effectiveness of the motor point block. In addition, the results of our study could be available directly if fixing were difficult as in the block for young patients or asses to EMG equipment were impossible. It is thought that the future studies based on the results of our study, about the specific way of botulinum toxin injection and the subsequent difference of the actual clinical efficacy, will be needed.

The biggest limitation with respect to our experiment is that we could not totally exclude the likelihood that the volume conduction of the deep muscles had contributed to the generation of the compound muscle action potential. In such regard, the future research through the analysis of the waveform of biceps brachii and if possible, including brachialis, is thought to be necessary. In addition, when obtaining the compound muscle action potentials by stimulating the main nerve, inter-trial varia- tion could happen. To reduce this possibility as much as possible, in our study, one author conducted a research, looking at the aspects of the waveforms from the beginning to the end of the test. However, complete exclusion was thought to be impossible. For this, future research using multi-channel recording, etc. should be considered. Further, in the actual clinical application, if considering that the biceps brachii is a superficial muscle, using good resolution ultrasound could be useful.

In addition to motor point block, the results of our study could be very useful for setting insertion site of needle electrode in electromyography, specifying the location of the electrodes in electrical stimulation and performing motor point muscle biopsy in the disease such as myasthenia gravis.

\section{CONCLUSION}

In this study, we conducted surface mapping of the electrophysiological motor point of biceps brachii muscle and concluded as follows.

When the vertical length of the upper arm, defined as the length of the line connecting coracoid process and mid-point of the elbow crease along with the horizontal length as the length of the elbow crease, the motor point of short head and long head of biceps brachii were located at about $2 / 3$ of the vertical length from the coracoid process, respectively, and about $1 / 5$ of the half of the horizontal length from the vertical reference line on both sides. Further, the motor point of the short head was located more medially and distally in the male compared with that in the female, but there was no difference in the motor point of the long head.

The information of the motor point of biceps brachii muscle in this study is thought to be very useful to improve therapeutic effect of spasticity treatment by allowing botulinum neurotoxin to be injected near to motor endplate. Putting together the results of this study and the cadaver study, it is recommended that the definition of motor point and clinical approach need to be varied, according to which block is performed in the phenol or botulinum neurotoxin. In addition, the results of this study are considered to be highly useful information for electromyography test, electrical stimulation, and even motor point muscle biopsy. 


\section{ACKNOWLEDGEMENTS}

We thank God and greatly appreciate the commentary assistance of Hyun-Yoon Ko, Byung Kyu Park and Chang hwan Kim and Gyu-Young Chai and are indebted to Young-Dae Kim for his skillful technical assistance.

\section{REFERENCES}

1. Bang MS, Han TR, Lim JY. Motor point block by phenol in spastic cerebral palsy. J Korean Acad Rehab Med 1997; 21: 71-77

2. Rousseaux M, Kozlowski O, Froger J. Efficacy of botulinum toxin A in upper limb function of hemiplegic patients. J Neurol 2002; 249: 76-84

3. Shaari CM, Sanders I. Quantifying how location and dose of botulinum toxin injections affect muscle paralysis. Muscle Nerve 1993; 16: 964-969

4. Childers MK, Kornegay JN, Aoki R, Otaviani L, Bogan DJ, Petroski G. Evaluating motor end-plate-targeted injections of botulinum toxin type $A$ in a canine model. Muscle Nerve 1998; 21: 653-655

5. Awad EA, Dykstra D. Treatment of spasticity by neurolysis. In: Kottke FJ, Lehmann JF, Editors. Krusen's handbook of physical medicine and rehabilitation, 4th ed, Philadelphia: Saunders, 1990, 1154-1161

6. Dumitru D. Nerve and muscle anatomy and physiology. In: King JC, Robinson LR, Spielholz NI, Turk MA, Editors. Electrodiagnostic medicine,1st ed, Philadelphia: Henley \& Belfus Inc, 1995, 19

7. Kim HS, Lee KW, Kim JM, Chung SH, Kim SY. Localization of the motor nerve branches and motor points of the hamstring muscles and triceps surae muscle. J Korean Acad Rehab Med 1998; 22: 1305-1311

8. Kim MW, Kim JH, Ko YJ, Moon JS, Yang YJ. Anatomical locations of the motor points of the triceps surae muscles. J Korean Acad Rehab Med 2003; 27: 581-584

9. Buchanan TS, Erickson JC. Selective block of the brachialis motor point. An anatomic investigation of musculocutaneous nerve branching. Reg Anesth 1996; 21: 89-92

10. Kim JS, Kwon JY, Kang SY, park JW. Anatomical locations of the motor points of the biceps brachii and brachialis muscles. J Korean Acad Rehab Med 2004; 28: 592-595

11. Park BK, Shin YB, Ko HY, Park JH, Baek SY. Anatomic motor point localization of the biceps brachii and bra- chialis muscles. J Korean Med Sci 2007; 22: 459-462

12. Lee JH, Kim HW, Im S, An X, Lee MS, Lee UY, Han SH. Localization of motor entry points and terminal intramuscular nerve endings of the musculocutaneous nerve to biceps and brachialis muscles. Surg Radiol Anat 2010; 32: 213-220

13. Keenan MA. Management of the spastic upper extremity in the neurologically impaired adult. Clin Orthop Relat Res 1988; 233: 116-125

14. Keenan MA, Tomas ES, Stone L, Gersten LM. Percutaneous phenol block of the musculocutaneous nerve to control elbow flexor spasticity. J Hand Surg [Am] 1990; 15: 340-346

15. Bang IK, Kim C, Ahn JK, Park YK, Reu HW, Jung IT. Sonographically guided musculocutaneous nerve phenol block for elbow flexor spasticity: case report. J Korean Acad Rehab Med 2007; 31: 371-374

16. Chin TY, Duncan JA, Johnstone BR, Graham HK. Management of the upper limb in cerebral palsy. J Pediatr Orthop B 2005; 14: 389-404

17. Gracies JM, Lugassy M, Weisz DJ, Vecchio M, Flanagan S, Simpson DM. Botulinum toxin dilution and endplate targeting in spasticity: a double-blind controlled study. Arch Phys Med Rehabil 2009; 90: 9-16

18. Mayer NH, Whyte J, Wannstedt G, Ellis CA. Comparative impact of 2 botulinum toxin injection techniques for elbow flexor hypertonia. Arch Phys Med Rehabil 2008; 89: 982-987

19. Francisco GE, Boake C, Vaughn A. Botulinum toxin in upper limb spasticity after acquired brain injury. A randomized trial comparing dilution techniques. Am J Phys Med Rehabil 2002; 81: 355-363

20. Ko HY, Park HJ, Park JH, Kim H. Surface mapping of motor points of gastrocnemius and soleus muscles. J Korean Acad Rehab Med 2001; 25: 621-626

21. Aquilonius SM, Askmark H, Gillberg PG, Nandedkar S, Olsson Y, Stalberg E. Topographical localization of motor endplates in cryosections of whole human muscles. Muscle Nerve 1984; 7: 287-293

22. Amirali A, Mu L, Gracies JM, Simpson DM. Anatomical localization of motor endplate bands in the human biceps brachii. J Clin Neuromuscul Dis 2007; 9: 306312

23. Coërs C. Structural organization of the motor nerve endings in mammalian muscle spindles and other striated muscle fibers. Am J Phys Med 1959; 38: 166175 
24. Christensen E. Topography of terminal motor innervation in striated muscles from stillborn infants. Am J Phys Med 1959; 38: 65-78

25. Deshpande S, Gormley ME, Carvey JR. Muscle fiber orientation in muscles commonly injected with botulinum toxin: an anatomical pilot study. Neurotox Res 2006; 9: 115-120

26. Masuda T, Miyano H, Sadoyama T. The distribution of myoneural junctions in the biceps brachii investigated by surface electromyography. Electroencephalogr Clin Neurophysiol 1983; 56: 597-603

27. Saitou K, Masuda T, Michikami D, Kojima R, Okada M. Innervation zones of the upper and lower limb muscles estimated by using multichannel surface EMG. J
Hum Ergol (Tokyo) 2000; 29: 35-52

28. Chiarapattanakom $P$, Leechavengvongs S, Witoonchart K, Uerpairojkit C, Thuvasethakul P. Anatomy and internal topography of the musculocutaneous nerve: the nerves to the biceps and brachialis muscle. J Hand Surg Am 1998; 23: 250-255

29. Gaertner E, Kern O, Mahoudeau G, Freys G, Golfetto $\mathrm{T}$, Calon B. Block of brachial plexus branches by the humeral route. A prospective study in 503 ambulatory patients. Proposal of a nerve-blocking sequence. Acta Anaesthesiol Scand 1999; 43: 609-613

30. O'Brien CF. Injection techniques for botulinum toxin using electromyography and electrical simulation. Muscle Nerve Suppl 1997; 6: S176-180 